\title{
Stilwechsel und Bewußtseinswandel
}

\author{
Von Peter Anselm Riedl
}

Die Umstände machen eine Vorbemerkung nötig. Die Veranstaltung sollte eigentlich in der Aula der Alten Universität stattfinden, und ich wollte in meiner Argumentation von dem Gemälde ausgehen, das Sie hätten vor sich haben und unter dem ich hätte reden sollen. Jetzt muß ich Sie also bitten, sich in der Vorstellung in die Alte Aula, die Sie ja alle gut kennen, zu begeben und sich dabei von ein paar Diapositiven helfen zu lassen.

Das Lünettenbild an der Stimwand dieser Aula ist wie die gesamte sichtbare Ausstattung des Saales 1886 entstanden. Damals ließ man anläßlich der Fünfhundertjahrfeier unserer Universität die barocke Dekoration kurzerhand hinter einer historistischen Auskleidung verschwinden; die Stukkaturen und Deckenmalereien des frühen achtzehnten Jahrhunderts sind in der Tat noch teilweise unter der jüngeren Schicht verborgen und könnten jederzeit freigelegt werden -

* Marsilius-Vortrag, gehalten im Rahmen der Jahresfeier der Ruprecht-Karls-Universität am 19. Oktober 1996 in der Aula der Neuen Universität in Heidelberg. Die seinerzeit vorgeführten Bildbeispiele müssen hier auf zwei programmatisch kontrastierende Abbildungspaare verringert werden. Bewußt werden auch die folgenden Literaturhinweise beschränkt, und zwar auf solche zur Aula in der Alten Universität und zum Gemälde Ferdinand Kellers in diesem Saal (1) sowie solche zur Diskussion über die Anfänge der Gotik (2).

Ad 1: Michael Koch: Ferdinand Keller (1842-1922), Leben und Werk, Karlsruhe 1978. - Ulrike Grammbitter: Joseph Durm 1837-1919, Eine Einführung in das architektonische Werk, Diss. Heidelberg, München 1984. - Sabine Juschka: Die Alte Universität, in: Die Gebäude der Universität Heidelberg, Bände 5 und 6 der Festschrift zur Sechshundertjahrfeier der Universität Heidelberg, herausgegeben von Peter Anselm Riedl, Heidelberg 1985. - Sabine Juschka: Die künstlerische Gestaltung der Heidelberger Universitätsjubiläen, Veröffentlichungen zur Heidelberger Altstadt, herausgegeben von Peter Anselm Riedl, Heft 28, Heidelberg 1993. - Helmut Prückner. Eine Nike zum Fest, in: Lebendige Antike, Rezeptionen der Antike in Politik, Kunst und Wissenschaft der Neuzeit, Mannheimer Historische Forschungen, Band 6, Mannheim 1995, S. 187-194.

Ad 2: Christoph Markschies: Gibt es eine „Theologie der gotischen Kathedrale“? Nochmals Suger von Saint-Denis und Sankt Dionys vom Areopag. Abhandlungen der Heidelberger Akademie der Wissenschaften, Philosophisch-historische Klasse, Jahrgang 1995, 1. Abhandlung, Heidelberg 1995 (dort Bibliographie, S. 70-80). 
eine Möglichkeit, an die natürlich niemand ernsthaft denkt. Die kunstwissenschaftliche Rehabilitierung von Barock und Rokoko bahnte sich in den achtziger Jahren des neunzehnten Jahrhunderts erst an, aber die historistische Kunst hatte den Stil vielerorts bereits so adaptiert, wie sie sich vorher ältere Stile anverwandelt hatte. Doch zu einer Zeit, als in Frankreich die Pariser Grand Opéra stand, das Deuxième Rococo blühte und in Bayem Schloß Linderhof im Entstehen war, zerschlug man in Heidelberg das zweifellos bedeutendste spätbarocke Ausstattungsstück der ganzen Region, Paul Egells Hochaltar der Jesuitenkirche, und erdachte für das Innere dieses Baus einen altertümelnden Phantasiedekor.

Auch mit der barocken Aula mochte man sich nicht mehr abfinden. Josef Durm, sicherlich einer der profiliertesten Architekten des späten Historismus, machte aus dem hellgestimmten Raum ein Interieur, dem mehr das Flair des Alt-Ehrwürdigen eignet. Die Mischung von Stilformen der Renaissance, des Manierismus und des Frühbarocks hat gewiß ihre eigenen Reize, aber daß sie sich gegen die als künstlerisch unwürdig diffamierte Ausstattung des achtzehnten Jahrhunderts richtete, ist ebenso wenig zu verkennen wie die Tatsache, $\mathrm{da} ß$ dieses Jahrhundert auf dem großen Wandgemälde programmatisch übergangen ist. Auf dem Bilde sind nämlich einerseits Repräsentanten des Heidelberger Geisteslebens aus frühen Zeiten zu sehen, so Johann von Dalberg, Marsilius von Inghen, Rudolf Agricola, Sebastian Münster und Samuel Pufendorf, andererseits herausragende Professoren des neunzehnten Jahrhunderts, wie Friedrich von Schlosser, Anton Friedrich Thibaut und Maximilian Chelius; ausgeblendet sind die Vertreter des für Heidelbergs Universität wenig ruhmreichen achtzehnten Jahrhunderts.

Das Gemälde, über das in den letzten Jahren von verschiedenen Autoren wohl alles Wichtige gesagt worden ist, vergegenwärtigt die Stiftung der Universität durch Ruprecht I. im Jahre 1386. Pallas Athene, die Göttin der Weisheit und der Künste, hält in Heidelberg auf einem von zwei Schimmeln gezogenen Prunkwagen Einzug, geleitet von der Siegesbringerin Nike, die sich aus dem Bild heraus uns, den Betrachtern, zuwendet und uns so ins Geschehen hineinzieht. Kurfürst Ruprecht sitzt in der Mitte auf hohem, säulenflankierten Thron, assistiert von der Stadtgöttin Heidelbergs. Rechts führen Studenten in altdeutscher Tracht mit den Fahnen des Deutschen Reiches und des badischen Herrscherhauses den Festzug an. Links drängt sich die schon erwähnte Schar der Zelebritäten, angeführt von Johann von Dalberg im Bischofsornat. Rechts oben ist die Ruine des Heidelberger Schlosses zu sehen.

Es geht mir hier nicht um eine vollständige Beschreibung und Interpretation des Bildes, vielmehr um die Verdeutlichung des für ihn geltenden künstlerischen Prinzips. Wenn man dieses als historisch-allegorisches Prinzip bezeichnet, muß man gleich hinzufügen, daß Historie hier nicht als ein in sich stimmiges Ereignis aufgerufen ist: Sie wird ganz im Gegenteil durch Geschichtsaus- 


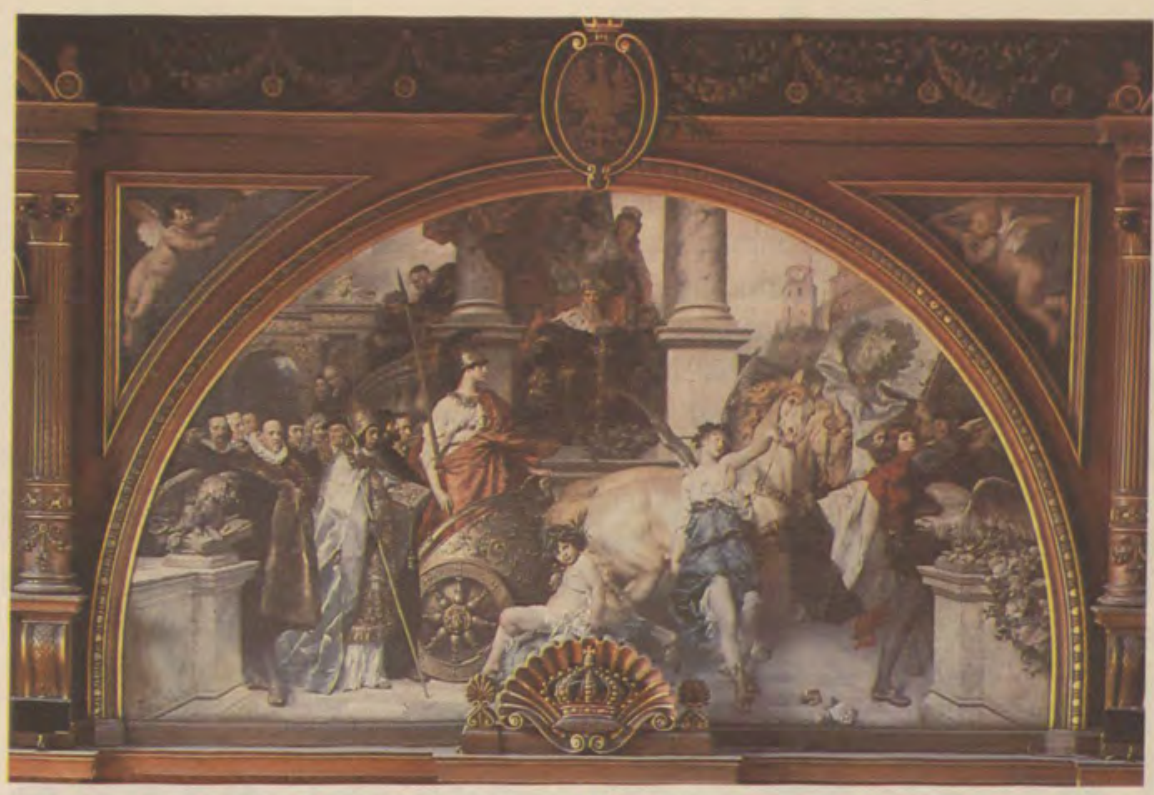

Abb. 1. Ferdinand Keller: Historische Allegorie der Gründung der Universität Heidelberg, 1886. Heidelberg, Aula der Alten Universität

schnitte vermittelt, die durch die Fiktion der Gleichzeitigkeit von Ungleichzeitigem einem höheren Darstellungsziel dienstbar gemacht sind. Die Personen gehören unterschiedlichen Geschichtsphasen an, die mittelalterliche Gründungsszene findet unter einer Schloßruine statt, die es erst seit dem Orléansschen Krieg gibt, Gelehrte des fünfzehnten und sechzehnten Jahrhunderts folgen der Flagge des Bismarckschen Reiches und so fort. Was solche Ungereimtheiten bühnenfähig macht, ist die verbindende Kraft der Allegorie. Ganz selbstverständlich setzt sie der Künstler des späten neunzehnten Jahrhunderts noch als das ein, was sie viele Jahrhunderte hindurch für die abendländische Kunst war: ein Element, das Verstreutes in ein Amalgam verwandeln konnte. Unentbehrlich war dabei die Welt der Mythologie, denn sie gab die Chance, Inhalte und Zusammenhänge zu versinnlichen, die sich einer historisch plausiblen Darstellung entzogen. Renaissance, Manierismus und Barock machten von dem hier skizzierten Modus ausgiebig Gebrauch - die Szene der Guten Regentschaft der Maria de' Medici aus Rubens' Luxembourg-Zyklus von 1621-25 mag es exemplarisch belegen. Und der Klassizismus bestätigte die fortdauernde Wirksamkeit des Prinzips in Gemälden wie Ingres' „Apotheose Homers"von 1827.

Auf Raffael, der bei Ingres als Kronzeuge des Klassischen im Kreise erlauchter Geister aus drei Jahrtausenden nicht fehlen darf, bezieht sich auch der Maler des Bildes unserer Aula. Schon bevor Ferdinand Keller, Professor für 
Historienmalerei an der Karlsruher Akademie, eingeschaltet wurde, hatte Josef Durm dazu geraten, die Figuren der von ihm vorgeschlagenen Darstellung nach dem Vorbild von Raffaels „Borgobrand“ in den vatikanischen Stanzen zu proportionieren. Keller beteuert wenig später seinerseits: „Diesen Größenbemessungen bin ich im neuen Entwurfe thunlichst nachgegangen und würde das Bild etwa dem sog: Borgobrand Raffaels nahekommen, bei dem die Wasserträgerin $1,80 \mathrm{~m}$ mißt und Form und Größe sich annähernd decken in beiden". Es sind auf den ersten Blick Äußerlichkeiten, die das historistische Produkt mit dem Werk Raffaels verknüpfen. Aber das CEuvre Kellers liefert genügend Beweise für Traditionsrekurse auch anderer Art. Der - gelegentlich als „badischer Makart" titulierte - Künstler fühlte sich stilistisch besonders der Malerei der italienischen Renaissance verpflichtet, dazu der Kunst Anselm Feuerbachs, die ihrerseits stark durch das Vorbild der venezianischen, florentinischen und römischen Renaissancemalerei geprägt ist. Mag das Epigonenhafte bei Keller überwiegen - der Atem für die effektvolle Inszenierung und die Virtuosität im Umgang mit dem Pinsel sind ihm nicht abzusprechen. Gerade in der Wiedergabe des Stofflichen erreicht Keller eine bemerkenswerte Meisterschaft, und ebenda konnte ihm Feuerbach ein Vorbild sein.

Als Ferdinand Keller an seiner Heidelberger Lünette arbeitete, malte ein fast gleichalteriger Zunftgenosse im provenzalischen Gardanne eine Landschaft, die viel weniger durch die Genauigkeit der Sachschilderung überzeugen will als durch die Art der Evidenzstiftung mittels Formen, die aus der Farbe heraus gewonnen sind. Dichte Gruppen von Pinselstrichen in Orangeocker, Rötlichgrau, Hellviolett und Helltürkis, dazu Grün in einigen Helldunkelstufungen formieren auf der Leinwand ein Gefüge, das nachdrücklich auf seinem Bildcharakter behartt. Das Flächige der Bilderscheinung behauptet sich gegen die Tiefe des Landschaftsraumes, diffuses Licht saugt die Plastizität der Hügel und der fernen Montagne Sainte-Victoire in sich auf, das auch im Himmel präsente Grün verwebt alle Zonen miteinander. Die Regeln der Mimesis, der getreuen Naturnachahmung, wie sie seit der Renaissance für die bildenden Künste verbindlich waren, haben sichtlich ausgedient. Nicht mehr die Illusionierung von stofflicher Wirklichkeit, sondern die Ersetzung von Stofflichkeitsanmutung durch den Eindruck einer Malerei mit betontem Eigenanspruch ist angesagt. Kunst hat, um die Formulierung des Autors der Provence-Landschaft aufzugreifen, ,parallel zur Natur“" zu gestalten - also nicht nach der Natur.

Paul Cézanne, um den es hier geht, war ganz und gar kein Traditionsfeind. Er hat sich immer wieder mit den großen Malern der Vergangenheit auseinandergesetzt, und Maurice Denis hat ihm sogar unterstellt, daß er „aus dem Impressionismus etwas Solides und Dauerhaftes wie die Kunst der Museen machen" wollte. Gleichwohl hat Cézanne sich entschieden von den Vorstellungen der offiziellen - das heißt der akademischen - Kunst der Epoche abgewendet und damit zusammen mit einigen anderen eine Entwicklung eingeleitet, die in 


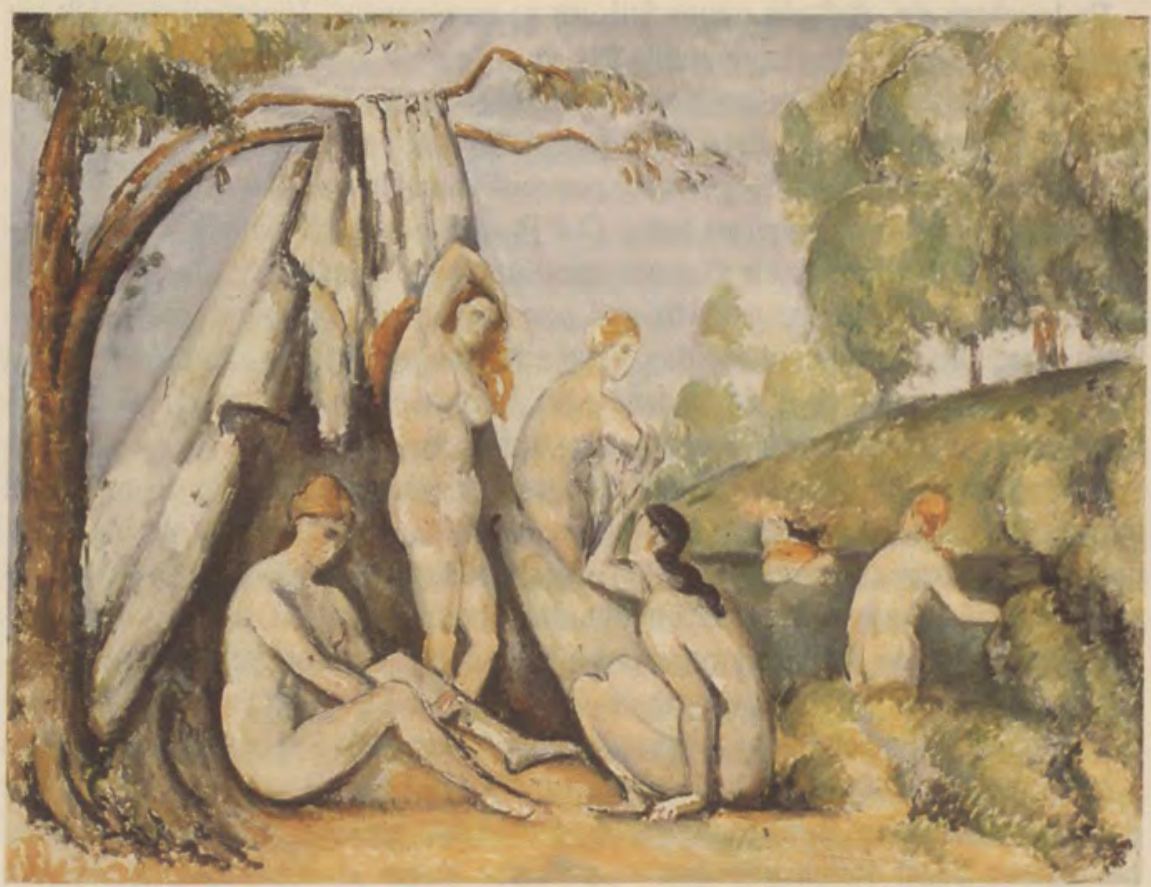

Abb. 2. Paul Cézanne: Weibliche Badende vor einem Zelt, 1883/85. Stuttgart, Staatsgalerie

die frühe Moderne führt. Schon in den achtziger Jahren des neunzehnten Jahrhunderts hat er Bilder wie „Weibliche Badende vor einem Zelt“" gemalt, in denen anatomische und perspektivische Richtigkeit einer strukturellen Logik geopfert erscheinen, die eine primär künstlerische ist.

Jede und jeder an der Sache Interessierte wird, nach den malereigeschichtlich wichtigen Persönlichkeiten der achtziger Jahre des neunzehnten Jahrhunderts gefragt, sogleich an Cézanne denken, dazu an Vincent van Gogh, Paul Gauguin, Georges Seurat, Henri Toulouse-Lautrec und einige andere Angehörige der sogenannten postimpressionistischen Generation - schwerlich an Künstler von der Art eines Ferdinand Keller oder Anton von Werner. Das allgemein gültige wissenschaftliche Idealkonstrukt will, daß Geschichte das Wissen vom Wirken ihrer Pioniere ist. Tatsächlich ereignete sich das, was die Pioniere leisteten, zunächst in der Abgeschiedenheit von Insiderkreisen. Es gab nur wenige, die an Cézanne glaubten; van Gogh starb vereinsamt und unverstanden. Die Zeit kam für diese Meister erst im frühen zwanzigsten Jahrhundert. Als sie aus dem Zwielicht der Umstrittenheit herauszutreten begannen, hatte der Historismus als offizielle Kunst vielerorts noch immer nicht abgedankt. 
Es leuchtet ein, daß ein neuer Stil um so weniger auf Verständnis stößt, je mehr er Gewohntes in Frage stellt. Die vom Impressionismus und noch mehr vom Postimpressionismus vollzogene Abkehr von traditionellen Gestaltungsund Inhaltsvermittlungsweisen kam einem Paradigmenwechsel gleich, wie es ihn so einschneidend seit Beginn der neuzeitlichen Kunst, also seit der Frührenaissance, nicht mehr gegeben hatte. Der Prozeß der Verabschiedung des Hergebrachten selbst verlief als Ganzes insofern eher pragmatisch, als er von keiner einheitlichen Theorie gestützt und von keinem übergreifenden Willen gelenkt war. Die Argumente für das Neue entsprangen weithin dem Widerstand gegen das Alte; und weil sie in der Regel Argumente zugunsten des Subjektiven waren, entziehen sie sich synthetischer Beschreibung.

So selbstverständlich es ist, daß es für Stilwechsel bestimmte Gründe und Rahmenbedingungen geben muß: Ist es vorstellbar, daß ein Stil als Innovationskonzept vorgedacht und punktuell durchgesetzt werden kann, um sich dann eigendynamisch zu verbreiten? Es gibt einen berühmten Fall, der in der Kunstgeschichte als von Autoritäten geklärt und in den Rang von Schulwissen erhoben schien, bis er kürzlich wieder ins Zentrum der Diskussion rückte. Seit Erwin Panofskys Forschungen in den vierziger Jahren galt Suger von Saint-Denis - er lebte von 1080/81 bis 1151 und war von 1122 bis zu seinem Tod Abt der damals wichtigsten Benediktinerabtei des Frankenreichs, in den letzten Lebensjahren sogar Reichsverweser -, galt also Suger als Schlüsselfigur in der Entstehungsgeschichte der Gotik. In zwei Bauabschnitten hatte Suger die karolingische Abteikirche um eine Zweiturmfassade und einen Chor mit doppeltem Umgang und Kapellenkranz bereichert; ein geplantes Langhaus blieb unausgeführt. Die (durch spätere Umbauten stark veränderten) Sugerschen Teile von Saint-Denis repräsentieren stilistisch in ganzer Klarheit die Frühphase der Gotik. In drei Schriften Ordinatio, De Consacratione und De Administratione berichtet Suger über den Neubau und seine künstlerische Ausschmückung; von den Chorkapellen heißt es da etwa, sie sollten „[...] den ganzen Raum in wunderbarem und gleichmäßig die Schönheit des Inneren durchleuchtendem Glanze ihrer Glasfenster mit den heiligsten Darstellungen erstrahlen lassen“. Auch mehrere Bauinschriften machen Aussagen über die der Architektur und den Glasmalereien anvertrauten Intentionen.

Sugers wiederholter Verweis auf Lichtphänomene brachte Panofsky auf einen fesselnden Gedanken: der Abt habe sich von den Schriften jenes christlich-neuplatonischen Mystikers aus dem Syrien des fünften Jahrhunderts anregen lassen, den die Tradition mit dem Areopagiten Dionysius gleichsetzte, einem biblischen Heiligen, der nun seinerseits in Saint-Denis mit dem hier bestatteten frühchristlichen Pariser Bischof und Märtyrer Dionys identifiziert wurde. Die Lichtmetaphysik des syrischen Pseudo-Dionysius sei, so Panofsky, als theologische Quelle der gotischen Architektur aufzufassen, Abt Suger sei Inspirator des neuen Stils. Otto von Simson spitzte in seinem Buch über die 
gotische Kathedrale 1956 Panofskys Thesen noch zu. Und in der Folge konnte man dann beispielsweise bei Christian Beutler 1970 über Suger lesen: „Der von ihm in Angriff genommene Neubau der Kirche [...] wurde zum Schöpfungsbau eines neuen Stils, der Gotik, die für Jahrhunderte die Architektur Europas bestimmen sollte, und er ist bis heute mit dem Namen des Abtes als seines eigentlichen Urhebers verbunden geblieben, während die Namen der ausführenden Baumeister unbekannt sind" (1970).

Einwände gegen die Thesen Panofskys und seiner zahlreichen Gefolgschaft kamen zunächst von seiten der Architekturgeschichte. Man meldete Zweifel an Sugers Kompetenz im Hinblick auf das Gesamtkonzept von Saint-Denis an und erläuterte, daß der angebliche Stilgründungsbau „eine Synthese von älteren Architekturelementen unterschiedlicher Herkunft ist". Was die Bauhistoriker wahrscheinlich machten, wurde im vergangenen Jahr in einer als Abhandlung der Heidelberger Akademie der Wissenschaften erschienenen Schrift von Christoph Markschies erhärtet. Auf die Frage „Gibt es eine ,Theologie der gotischen Kathedrale“" (so auch der Titel der Untersuchung) kommt der Kirchenhistoriker zu der recht provokanten Antwort eines „Nein!“. Markschies legt dar, daß Suger „kein origineller theologischer Denker war, sondern an der entsprechenden Koine seiner Zeit partizipierte", daß die Lichtterminologie der Bauinschriften gängiges, rein topisch angewendetes Gedankengut war und daß es, alles in allem, keinen Beweis für eine Umsetzung theologischer Ideen in gebaute Strukturen gibt. Auch wenn man nicht alle Beweisschritte des Autors mitvollziehen möchte und wenn die Architekturhistorie noch Lücken im Wissen zu schließen hat - beim heutigen Forschungsstand verbietet es sich, die Gotik für das theoretisch beziehungsweise theologisch begründete Produkt eines Willens zu halten, den man personalisieren könnte. Der Stil ist offenkundig vielmehr Resultat einer um 1130/40 an mehreren Stellen des französischen Kronlandes einsetzenden „Baubewegung“(Suckale).

Und wie steht es mit der Genese der italienischen Renaissance? Das, was in den Jahren seit etwa 1415 von einigen wenigen Architekten, Bildhauern, Malern und Kunsthandwerkern in Florenz gewollt und verwirklicht wurde, gehört zu den erstaunlichsten Phänomenen der Kunstgeschichte. Der herrschende Stil der späten Gotik wurde mit aller Entschiedenheit verworfen, der neue Stil als eine Norm etabliert, die im Laufe des fünfzehnten Jahrhunderts in Italien, später in ganz Europa Anerkennung fand. Daß schon in der Frühphase der Renaissance Werke von größter stilistischen Konsequenz - wie Filippo Brunelleschis Alte Sakristei oder Donatellos Bronze-David - entstanden, zeugt vom hohen Grad der Bewußtheit, mit derder Stilwechsel durchgesetzt wurde.

Nun muß man sich vor Augen halten, daß das Neue zugleich ein Altes war, denn es war ja die Kunst der Antike, auf welche die Renovatio oder Rinascita zielte. Für eine solche Wiedergeburt gab es in Italien seit der Zeit eines Dante, Petrarca und Giotto günstige Bedingungen; gleich einer Tiefenschicht blieben 
sie das ganze vierzehnte Jahrhundert hindurch erhalten und konnten jetzt den neuen Stil als Fundamente unterfangen. Als ein schöpferischer Irrtum unter anderen erwies es sich, daß die Renaissancepioniere die romanische Architektur der Toskana, die wir heute unter dem Begriff Protorenaissance fassen, für antik hielten. Was im Zeichen der Idee einer Wiedergeburt zustandekam, war, rückschauend betrachtet, durchaus innovativ. Aber vom Ansatz her war es dazu bestimmt, einen historischen Stil zu rehabilitieren und zu aktualisieren.

Manierismus, Barock und Rokoko müssen als Evolutionsstufen der Renaissance aufgefaßt werden. $\mathrm{Da} B$ in ihnen die Werte des begründenden Stiles zum Teil bis an die Grenzen beansprucht oder gar auf den Kopf gestellt sind, spricht nicht gegen ihren prinzipiellen Verwandtschaftszusammenhang. So reich an Wandlungen sich das Formenrepertoire zwischen Brunelleschi und Balthasar Neumann, Donatello und Ignaz Günther, Masaccio und Giambattista Tiepolo darbietet - es entstammt einer geschichtlichen Wurzel. Negativ wird das durch die von der klassizistischen Theorie eingeführte und bis weit ins neunzehnte Jahrhundert hinein immer wiederholte Behauptung belegt, der Barock sei eine späte Verfallsvariante der Renaissance.

Der Klassizismus selbst hat durch eine Einzelpersönlichkeit eine starke und zeitlich genau fixierbare Prägung erfahren. Doch Johann Joachim Winckelmanns Lehre hat einen reformerischen Kern, denn sie bindet das Neue, das den Barock absichtsvoll korrigieren soll, wiederum an einen historisch vorgegebenen Stil, den des griechischen Altertums, zurück -- oder an das, was sich die Zeit darunter vorstellte. Wahr ist außerdem, daß bereits vor Winckelmann in den Künsten eine Tendenz zur klärenden Vereinfachung auszumachen ist. Sie ist zum Teil durch den Einfluß der frühen Aufklärung bedingt, hat aber wohl auch entwicklungsimmanente Gründe, insofern als auf Perioden extremer Komplizierung solche der Beruhigung zu folgen pflegen. Winckelmann gab der Tendenz gewissermaßen einen Inhalt, an dem sich Bewußtsein entfalten oder durch den sich Bewußtsein bestätigt sehen konnte.

Nach dem Klassizismus wird aus der europäischen Stillandschaft bekanntlich ein unübersichtliches Terrain. Die Romantik bildet keinen einheitlichen Modus aus, sondern stellt sich als ein Zugleich von Mitteilungsweisen einzelner Künstler und Künstlergruppierungen dar. Rekursive Züge finden sich neben realistischen, Ansätze zu einer hochgradig individuell gestimmten und dabei theorieverankerten Kunst (wie der von Philipp Otto Runge) neben Versuchen, zu einer unschuldig-religiösen Kunst zurückzukehren (man denke an die Nazarener). Breitenwirkung verschaffen sich die verschiedenen Varianten des Historismus. Sie sind es, die an den Akademien zu Ehren kommen - und leider bald auch zur Erstarrung. Gegen sie wenden sich seit der Mitte des neunzehnten Jahrhunderts der Realismus eines Courbet, seit den siebziger Jahren die den Realismus auf die Spitze treibende Malerei der Impressionisten und seit den achtziger Jahren die Kunst der Postimpressionisten. Allerdings ohne einen Pu- 
blikumserfolg, der sich mit dem der akademisch sanktionierten Kunst messen könnte. Wenn ich damit wieder das Ausgangsthema berühre, dann nicht, um bei ihm zu verharren, sondern um von ihm aus eine Perspektive in unser Jahrhundert hinein zu eröffnen.

Die Reaktion der Postimpressionisten auf den Traditionalismus und auf den Impressionismus selbst war, wie gesagt, uneinheitlich und als Ganzes nicht theoriegelenkt. Aber interessanterweise lassen sich gerade in der Generation der Postimpressionisten Bemühungen fassen, Stil als ein gewolltes und systematisch begründetes Novum einzuführen. Gegen den Vorläuferstil war - ob reformbetonend oder revolutionär, ob offen oder unausgesprochen - noch jeder neue Stil gerichtet; es hätte ihm ansonsten die für eine Alternative unverzichtbare Durchsetzungskraft fehlen müssen. Was, wenn ich recht sehe, in der Geschichte der Stilwechsel ganz neu ist, ist das in den achtziger Jahren des vergangenen Jahrhunderts zu beobachtende Zusammentreffen der Momente Vorbildlosigkeit, Verwurzelung in einer klaren Intention und Theoriebezogenheit. Der Divisionismus oder Pointillismus Georges Seurats, entwickelt in den Jahren zwischen etwa 1885 und 1891, ist ein Stil, der historisch wohl eine bis Chevreul und Delacroix zunückreichende Vorgeschichte hat, aber kein direktes Modell, und der sich junge Erkenntnisse der Physiologie und der Optik zunutze macht. Dem Rezeptionsvorgang auf der Netzhaut erwächst eine Analogie im gemalten Bild, das sich aus vielen Pünktchen tendenziell unvermischter Farbe zusammensetzt. Das Sehen selbst wird zum Thema der Malerei.

Für Cézanne und Van Gogh gelten die genannten Bedingungen nur eingeschränkt, weil die Theorieausstattung des jeweiligen individuellen Stils weniger schlüssig ist als im Falle Seurats. Der Jugendstil, der um 1900 in Europa und Amerika zur Blüte kommt, ist so etwas wie eine konzertierte Rebellion gegen den Historismus: in sich außerordentlich vielgestaltig, zwischen elitär und volkstümlich changierend, reich an theoretischen Positionen und dabei für das Handwerkliche aufgeschlossen. Das unerhört vielstimmige Stilszenarium der Jahrhundertwende entläßt aus sich die Bewegungen, die den eigentlichen Anfang der Moderne markieren: den Expressionismus und den Kubismus. Beide verstehen sich primär als antitraditionalistisch, beide sind im Ansatz untheoretisch (der vielzitierte Neukantianismus der Kubisten ist eine von den Künstlern bereitwillig übernommene externe Deutungstheorie).

Die nächsten Schritte des jetzt immer ungestümer verlaufenden Stilentstehungsprozesses führen zu Resultaten, die das erläuterte Mehrfachkriterium geradezu beispielhaft erfüllen. Vor allem die Begründer der abstrakten Kunst bewähren sich im zweiten Jahrzehnt des Jahrhunderts in Praxis und Theorie als Verfechter absolut neuartiger Möglichkeiten. Wassily Kandinsky findet zwischen etwa 1910 und 1913 zu einer gegenstandsunabhängigen Bildsprache, über deren Wirkungsweise und deren Sinn er in der programmatischen Abhandlung „Über das Geistige in der Kunst" aufklärt. Den ersten Anstoß zum 
Gegenstandsverzicht habe er, so Kandinsky rückblickend, von einem der Heuschober-Gemälde Claude Monets erhalten; das Sujet sei da bereits weitaus weniger bedeutsam als die Malerei und folglich im Prinzip entbehrlich. (Monets Heuschober-Serie entstand übrigens knapp fünf Jahre nach unserer sujetgesättigten Aula-Lünette.) - Wenig später als Kandinsky wird Piet Mondrian zum Erfinder und Propagandisten eines rigorosen nichtgegenständlichen Stils und entwickelt Kasimir Malewitsch ein bildnerisches Idiom mit einem auch verbal verkündeten Unbedingtheitsanspruch.

Abstraktion, Neo-Plastizismus, Suprematismus, Orphismus, Futurismus, Konstruktivismus, Rayonismus - wir sind mitten im Stilpluralismus, wie er für unser Jahrhundert zu einem selbstverständlichen Faktum geworden ist. Daß Stile sehr unterschiedlicher, ja gegensätzlicher Art nebeneinander existieren würden, hat bereits Kandinsky hellsichtig vorausgesagt; wie verwirrend breit das Spektrum am Ende des Säkulums sein würde, hat er nicht wissen können. Aus dem Problem des allgemeinen Bewußtseinswandels in der Folge eines Stilwechsels (dem selbstverständlich auf seiten der für den Wechsel Verantwortlichen immer schon ein Bewußtseinswandel vorausgegangen sein muß!), ist, was die Rezipienten angeht, das Problem der Bereitschaft und Fähigkeit geworden, Stilvielfalt und rasche Stilentstehung als Bewußtseinsinhalte zu akzeptieren. Als Zeitgenossen der Postmoderne erleben wir, daß auch das Historische und das Klassische zu Elementen eines aktuellen Stils gemacht werden können. Freilich nicht mit der von der langen Tradition der Mimesis getragenen Unbefangenheit des Historismus, sondern mit der Skepsis oder der analytischen Lust später Nachfahren, die aus demÜberliefenungskontinuum entlassen sind.

Ferdinand Keller läßt Nike - wie Helmut Prückner gezeigt hat: in gelehrter Anlehnung an die Nike des Paionios - als eine Vermittlerin zwischen dem im Bild Inszenierten und dem Publikum auftreten; er vertraut dabei noch auf die Gültigkeit von formalen und ikonographischen Konventionen, deren Fundamente in Wahrheit am Bröckeln sind. 1909 wird eine andere antike Nike als Zeugin einer überlebten Ästhetik aufgenufen. Im „Ersten Futuristischen Manifest" stehen die berühmten Sätze: „[...] wir behaupten, daß die Pracht der Welt sich um eine neue Schönheit bereichert hat: die Schönheit der Geschwindigkeit. Ein Rennwagen ist schöner als die Nike von Samothrake". Nicht mehr die Schönheit einer in sich geschlossenen Erscheinung ist gefragt, sondern eine Schönheit, die sich aus der sichtbaren Verschmelzung von Gegenstandsbewegung und Bewegtheit des umgebenden Raumes ergibt. Umberto Boccionis Bronze „Forme uniche della continuità nello spazio“ („Einzige Formen der Kontinuität im Raum") löst diese Erwartung ein, indem sie einen menschlichen Körper gleichsam den Energien des Raumes preisgegeben zeigt. Für die Futuristen besitzt die dynamistische Theorie den Rang eines Stilstiftungs-, ja eines Welterklärungsmodells. Doch dieses Modell konkurriert in seiner Zeit mit mehreren anderen, und es sollte, wie die anderen, der allgemeinen Dynamik der 


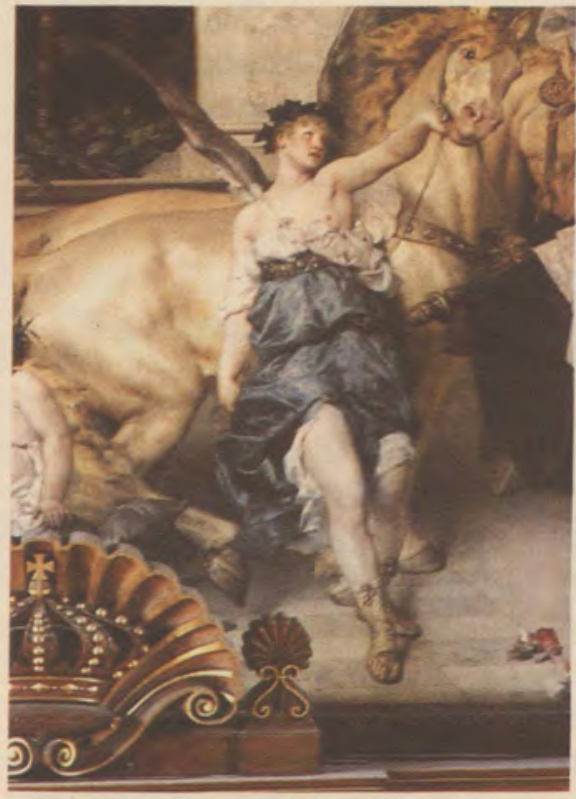

Abb. 3. Ferdinand Keller: Nike. Ausschnitt aus Abb. 1

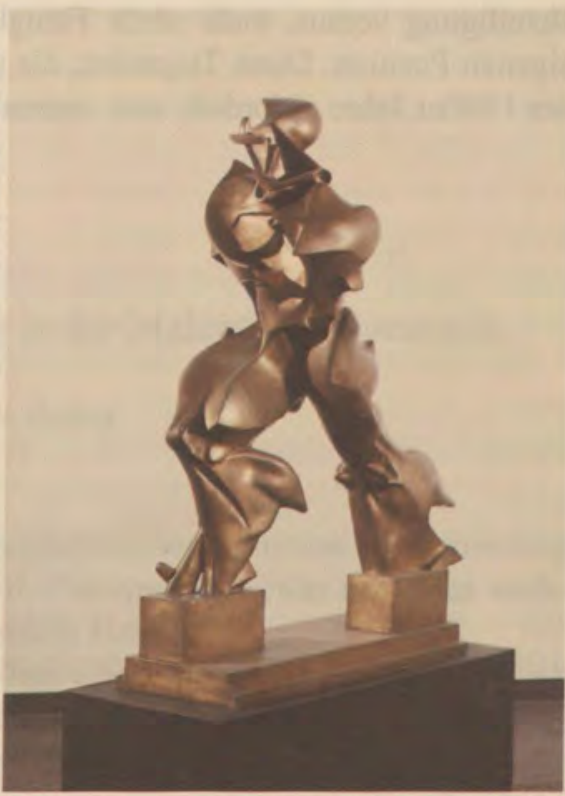

Abb. 4. Umberto Boccioni: Forme uniche della continuità nello spazio. 1913. Mannheim, Städtische Kunsthalle

Stilentwicklung nur wenige Jahre standhalten, um dann von neuen Modellen abgelöst zu werden.

Unsere Aula-Lünette hat uns zu ausgedehnten Erkundungen Anlaß gegeben. Der Historist Ferdinand Keller konnte gewiß nicht ahnen, daß er sein Bild in einem kunsthistorischen Schwellenmoment schuf. Mitte der achtziger Jahre des neunzehnten Jahrhunderts - und das wäre meine Kernaussage - gibt sich in Frankreich zum ersten Mal jener neue Typus von Stil zu erkennen, der ganz wesentlich den weiteren Gang der Kunstgeschichte prägen sollte: der Typus des von einer Einzelpersönlichkeit oder einer kleinen Gruppe getragenen, theorieverankerten und betont innovationsorientierten Stils. War Stilwechsel bis dahin ein Prozeß, der entweder eher gleitend verlief oder der, wenn er ein plötzlicher war, an älteren Vorbildern Rückhalt suchte, so wurde er jetzt zu einem Vorgang mit einem eigentümlichenUnbedingtheitsanspruch.

Und das Bewußtsein hatte sich auf diesen veränderten Stiltypus einzustellen. Bis dahin war die Aneignung von Neuem für das Publikum ein Vorgang mit einer gewissen Verzögerungstoleranz (auch wenn den Innovatoren solche Toleranz nicht eben lieb sein konnte). Jetzt blieb angesichts der Komplexität und der hohen Wechselfrequenz der Stile im Grunde nur die Wahl des Mitgehens oder der Verweigerung. Mitgehen setzt Offenheit und Bereitschaft zur inneren 
Beteiligung voraus, mehr noch: Fähigkeit zur unablässigen Überprüfung der eigenen Position. Diese Tugenden, die uns Kunst seit dem Paradigmenwechsel der 1880er Jahre abfordert, sind, meine ich, in unserer Welt unverzichtbar. 